\title{
Nanoscale gold pillars strengthened through dislocation starvation
}

\author{
Julia R. Greer and William D. Nix \\ Department of Materials Science and Engineering, Stanford University, 416 Escondido Mall, Stanford, California 94305, USA
}

(Received 21 April 2006; published 12 June 2006)

\begin{abstract}
It has been known for more than half a century that crystals can be made stronger by introducing defects into them, i.e., by strain-hardening. As the number of defects increases, their movement and multiplication is impeded, thus strengthening the material. In the present work we show hardening by dislocation starvation, a fundamentally different strengthening mechanism based on the elimination of defects from the crystal. We demonstrate that submicrometer sized gold crystals can be 50 times stronger than their bulk counterparts due to the elimination of defects from the crystal in the course of deformation.
\end{abstract}

DOI: 10.1103/PhysRevB.73.245410

PACS number(s): 62.25. $+\mathrm{g}$, 81.07.Bc, 81.16.Rf, 81.70.Bt

Anyone who has ever repeatedly bent a copper wire knows that it gets progressively stronger as it becomes more deformed, through a phenomenon called strain-hardening. The strengths of cold-worked metals are known to be up to ten times greater than those of well-annealed crystals. Plasticity in metals occurs by the motion of dislocations, or line defects, which multiply in the course of plastic deformation. Impeding the motion of dislocations by introducing defects into crystals results in strengthening. Although these fundamental concepts are often assumed to be applicable to crystals of any dimensions, numerous recent studies have shown that conventional plasticity diverges at a certain length scale, with smaller samples reported to be stronger than their bulk counterparts. $^{1-6}$

Pure metals and some alloys exhibit strong size effects at the submicron scale. ${ }^{1-13}$ Size effects in indentation, torsion, and bending have been understood in terms of the nonuniformity of the deformation, which sets up strain gradients leading to hardening. ${ }^{7}$ Size effects are also found in thin films, where the strength scales inversely with film thickness and is usually attributed to the confinement of dislocations by the substrate. ${ }^{8-10}$ Size effects are observed for pristine crystals, as well. ${ }^{11,12}$ In the earliest stages of nanoindentation, for example, the crystal volume is extremely small and can be dislocation-free, requiring very large stresses to nucleate new dislocations. In addition, classic experiments on the initially dislocation-free metal whiskers indicated that whiskers with smaller diameters yielded at higher stresses. ${ }^{13}$ In typical whiskerlike deformation behavior, initial elastic loading leads to a very high stress followed by a significant drop and continued plastic flow at low stresses. Finally, several molecular dynamics simulations ${ }^{14-16}$ and more recent experiments on small pillars ${ }^{17,18}$ all support the tenet that smaller is stronger. In spite of much progress on size effects research there is still no unified theory for plastic deformation at the submicron scale.

We focus on size effects arising in unconstrained geometries, in the absence of strain gradients, and with nonzero initial dislocation densities. Gold nanopillars ranging in diameter between $200 \mathrm{~nm}$ and several micrometers were fabricated using focused ion beam (FIB) machining and a microlithography/electroplating technique. These small pillars are found to plastically deform in uniaxial compression at stresses as high as $800 \mathrm{MPa}$, a value $\sim 50$ times higher than for bulk gold. We believe that these high strengths are controlled by the process of hardening by dislocation starvation. In this mechanism, once the sample is small enough, the mobile dislocations have a higher probability of annihilating at a nearby free surface than of multiplying and being pinned by other dislocations. When the starvation conditions are met, plasticity is accommodated by the nucleation and motion of new dislocations rather than by motion and interactions of existing dislocations, as is the case for bulk crystals.

The primary pillar fabrication method utilizes FIB machining to etch patterns of interest into single crystal gold. Following the approach developed by Uchic et al. ${ }^{17}$ and used by Greer et al. ${ }^{18}$ the present work extends this fabrication technique to much smaller nanopillars (Fig. 1).

We have also developed a uniaxial testing technique for studying mechanical properties of these tiny samples. Uniaxial compression tests on pillars of varying sizes and aspect ratios were conducted using the MTS Nanoindenter ${ }^{\mathrm{XP}}$ with a flat punch diamond tip. The tip was custom-machined from a standard Berkovich indenter by etching off the tip in the FIB. The resulting projected area is an equilateral triangle with a $9 \mu \mathrm{m}$ inscribed circle diameter. Unlike previously reported uniaxial compression studies, the loading mechanism here is nominally displacement-rate controlled rather than load-controlled. ${ }^{18}$ The "nominal displacement rate" here refers to the variable in the software method specified by the user. We wrote this method to simulate a constant displacement rate, and therefore a nearly constant strain rate. The method utilizes a PID-based feedback loop to control the displacement rate throughout the data acquisition in order to enable the instrument to quickly remove the imposed load during a discrete strain burst. Unfortunately, despite utilizing this approach, the Nanoindenter, which is inherently a loadcontrolled instrument, is not capable of responding to every slip event quickly enough. Therefore, the strain rate during the plastic components of the stress-strain curve cannot be fully controlled.

Load-displacement data were collected in the continuous stiffness measurement (CSM) mode of the instrument. Once the surface of the pillar has been detected, such parameters as load, harmonic contact stiffness, and compressive displacement of the top of the pillar are continuously measured and recorded. The load-displacement data obtained during the compression experiments are then converted to uniaxial stresses and strains using the assumption that the plastic volume is conserved throughout this mostly-homogeneous de- 

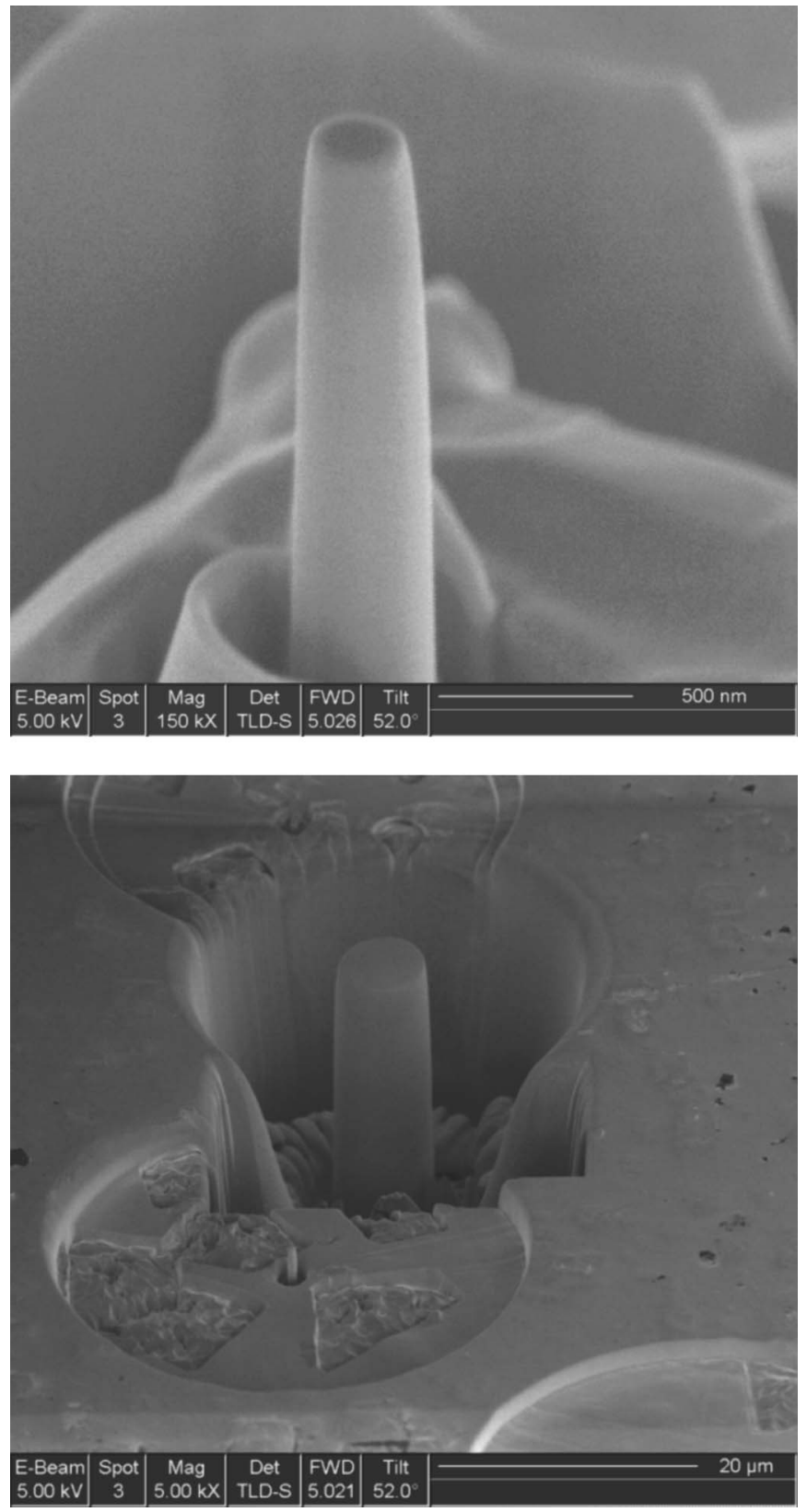

FIG. 1. (a) A representative $\langle 001\rangle$-oriented gold pillar machined in the FIB. Pillar diameter $=290 \mathrm{~nm}$, pillar height $=1.2 \mu \mathrm{m}$. (b) A large pillar $(7.45 \mu \mathrm{m}$ diameter) and a small pillar $(250 \mathrm{~nm}$ diameter).

formation. Stress-strain curves of FIB pillars whose diameters range between $290 \mathrm{~nm}$ and $7450 \mathrm{~nm}$ as well as the strength of bulk gold at 2\% strain are presented in Fig. 2(a). Uniaxial loading in the $\langle 001\rangle$ direction, chosen for our experiments and corresponding to a high-symmetry orientation, would result in the activation of 12 different $\{111\} /\langle 01 \overline{1}\rangle$ slip systems, with the pillar deforming uniformly around its diameter as it is compressed. In this orientation, despite the presence of the end constraints, the pillar remains centrallyloaded and preserves its cylindrical shape throughout the deformation process.

Each curve represents a single test at a specific pillar di- ameter, measured at approximately $L / 3$ below the pillar top. The smallest pillar reaches a compressive stress of $800 \mathrm{MPa}$ at $10 \%$ strain. While in some cases the initial stages of deformation are not purely elastic due to the gradual onset of yielding, the initial loading slopes of the well-aligned tests give elastic moduli very close to the Young's modulus of gold in the $\langle 001\rangle$ direction, $43 \mathrm{GPa}$. The fully elastic unloading slopes closely match the expected value, as well.

Another important aspect of these stress-strain curves is the lack of stage II work-hardening associated with the activation of multiple slip systems in the course of deformation of single crystals. In a typical single crystal, the dislocations from different active slip planes interact with each other and form sessile dislocations, creating a large number of barriers for the movement of other dislocations, thereby requiring ever-higher stresses with the increasing strain. A typical work-hardening slope is on the order of $\mu / 20$, where $\mu$ is the elastic shear modulus. This behavior is prevalent in the compression of the largest pillar whose diameter is a little over $7 \mu \mathrm{m}$, as shown in Fig. 3(a).

Contrary to this, the stress-strain behavior observed here agrees more with stage I-type deformation, or the "easy glide" section of a low-symmetry oriented single crystal deformation curve. Moreover, a representative stress-strain curve in this work is composed of a number of discrete slip events separated by elastic loading segments, while the overall stress level remains nearly constant as the strain increases as shown in Fig. 3(b). This suggests that the hardening mechanism here is the opposite to that of conventional strain-hardening, with the elastic loading sections indicative of the absence of dislocations rather than their multiplication.

One of the major concerns with the FIB fabrication technique is the possibility of $\mathrm{Ga}^{+}$ion implantation into the sample, and that this is the cause for the observed increase in strength. Several approaches were used to address this issue. First, Auger depth profiling analysis with subsequent surface layer removal was employed. The initial concentration of $\mathrm{Ga}$ was 1.7 at. $\%$ on top of the pillar and 0.8 at. $\%$ on its side. The "side" of the pillar here refers to the middle third of the specimen since in that area the deformation is closest to being homogeneous. The conformal surface layer was removed by etching the rotating pillar in low-energy $\mathrm{Ar}^{+}$plasma. Depth profiling and surface etching were repeated to assess the ever-decreasing concentration of $\mathrm{Ga}$. The final etch step resulted in the total removal of $5 \mathrm{~nm}$ from the pillar surface, reducing the overall $\mathrm{Ga}$ concentration by $\sim 50 \%$, and the gallium-to-gold ratio from 0.079 to 0.016 . The significant change in gold-to-gallium ratio indicates that $\mathrm{Ga}$ ions were located near the surface rather than implanted into the sample. These cleaned pillars were subsequently tested in compression, which indicated that surface removal had little effect on strength. The full Auger analysis can be found in Ref. 19(a).

To further investigate the possible effects of $\mathrm{Ga}^{+}$ion implantation, we developed an alternative fabrication technique based on lithography/electroplating. ${ }^{18}$ The electroplated pillars were annealed at $300{ }^{\circ} \mathrm{C}$ before testing to establish a coarse grain size. While these pillars are polycrystalline, they contain only 2-3 grains extending across the pillar width. To complete the analysis of possible surface modification, some 

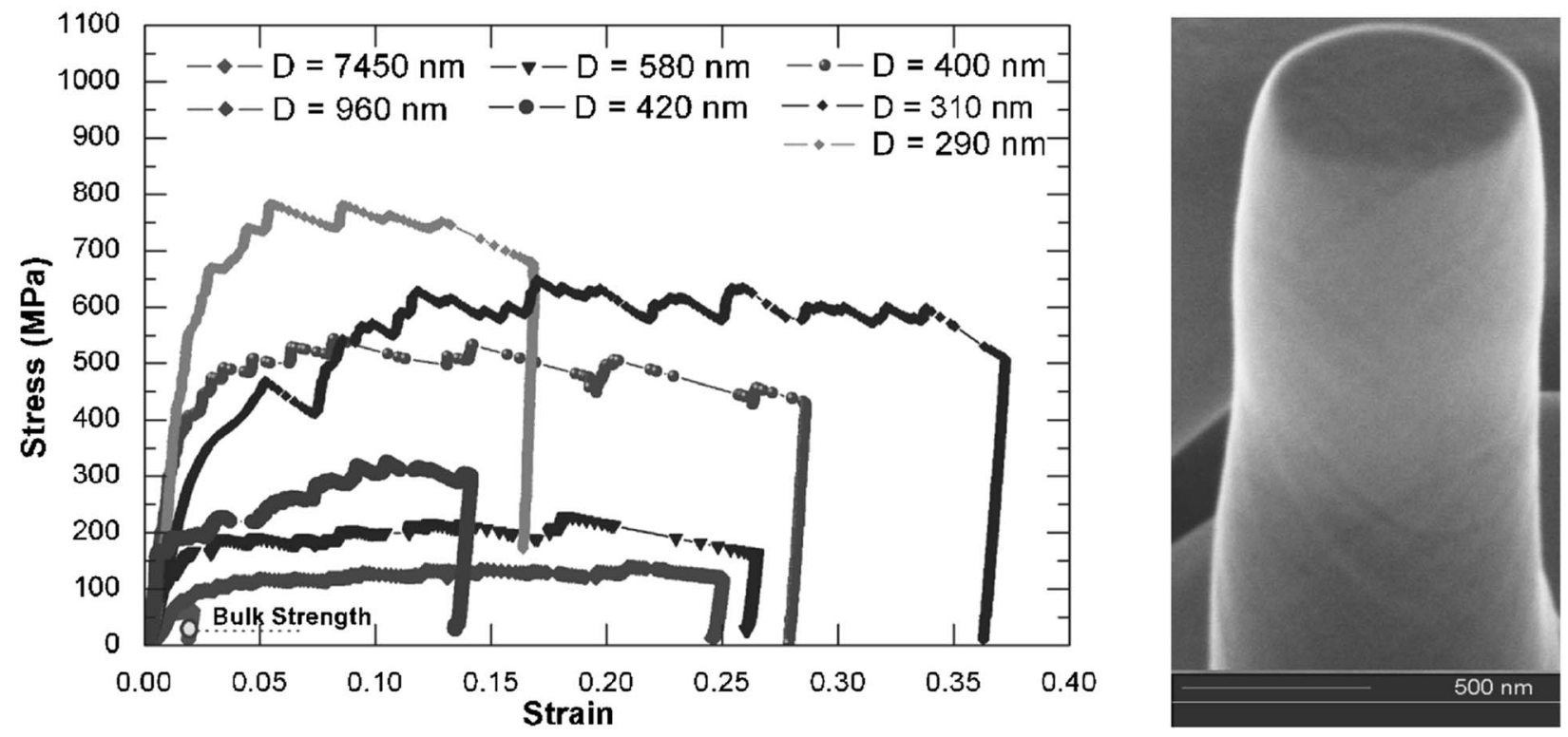

FIG. 2. (a) Stress-strain behavior of $\langle 001\rangle$-oriented pillars: flow stresses increase significantly as the pillar diameter is reduced. (b) SEM image of a compressed pillar after deformation. Slip lines in multiple orientations are clearly present and indicate a homogeneous shape change.
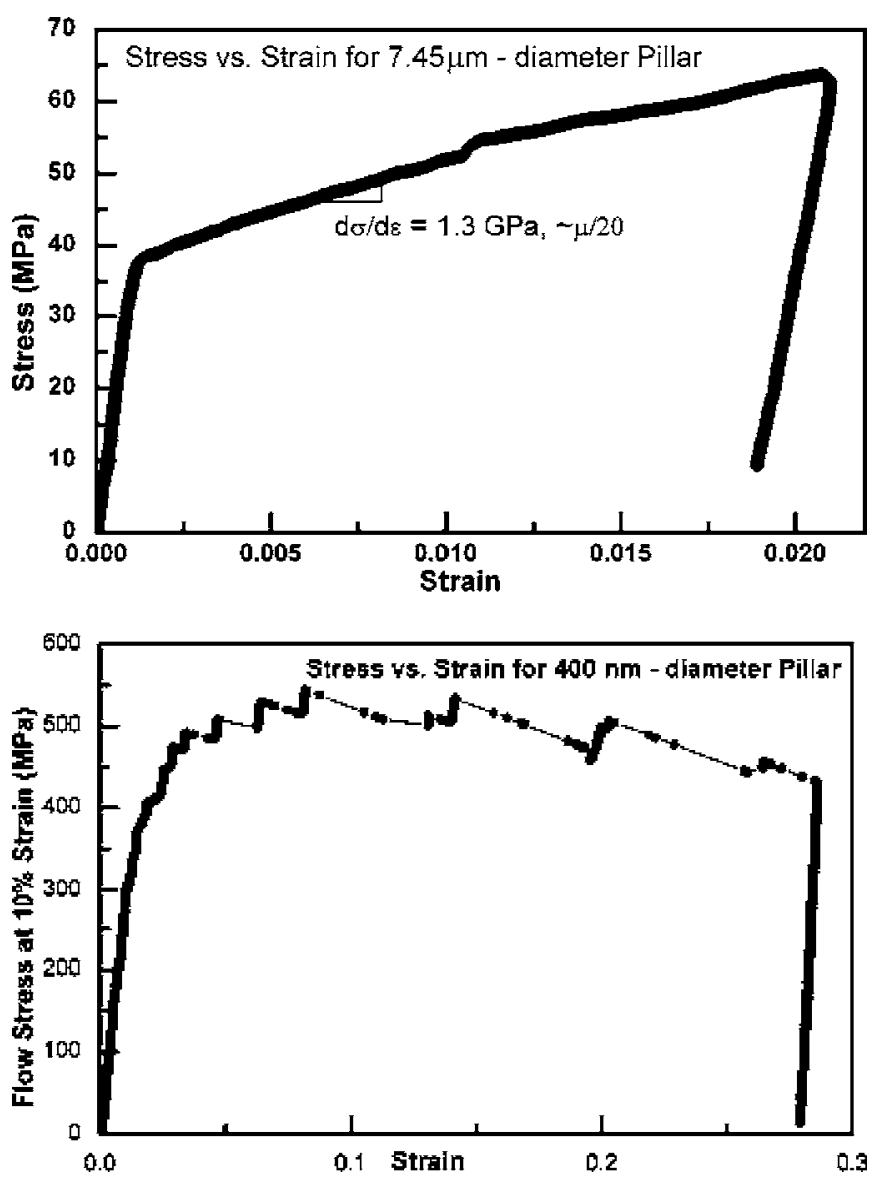

FIG. 3. (a) Stage II work-hardening is clearly present in the stress vs strain curve for the largest pillar whose diameter is $7.45 \mu \mathrm{m}$. (b) The lack of stage II work-hardening is evident in the stress vs strain curve for a small pillar whose diameter is $400 \mathrm{~nm}$. single crystal pillars were annealed at $300{ }^{\circ} \mathrm{C}$ to remove any remaining defects. Figure 4 presents flow stresses of all aforementioned samples as well as the FIB-fabricated samples and a comparison to the axial stress corresponding to the theoretical shear strength calculated by Ogata et al. ${ }^{20}$ All data points represent the pillars which deformed homogeneously during compression testing, as can be seen in Fig. 2(b).

It is clear from the graph that the electroplated pillars, which were never subjected to the $\mathrm{Ga}^{+}$ions, not only have flow stresses higher than bulk gold but also exhibit a similar rise in strength as the diameter is reduced. It is also clear that $\mathrm{Ga}^{+}$removal and pretest-annealing resulted in data that falls on the curve formed by the FIB pillars. This result leads us to believe that observed size effect is not linked to a specific fabrication technique. While some minimal $\mathrm{Ga}^{+}$might be

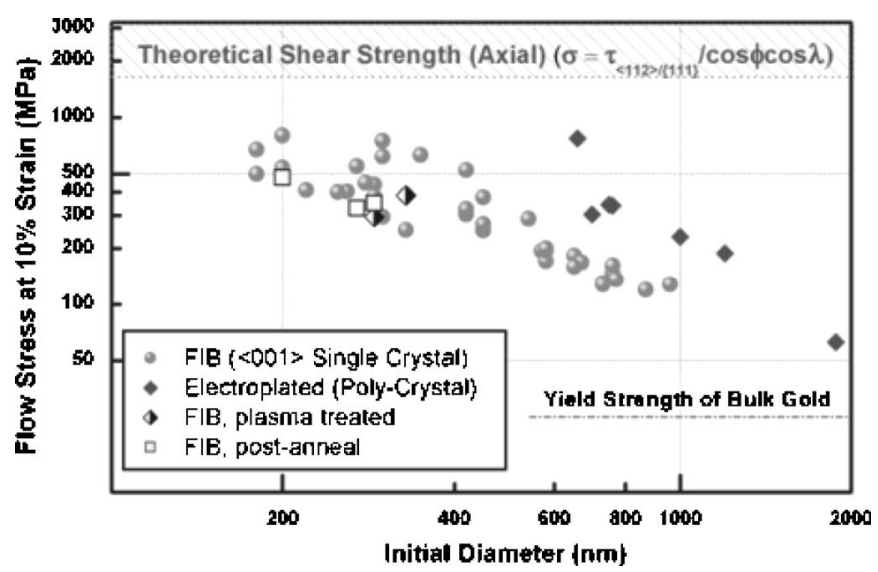

FIG. 4. Flow stress vs pillar diameter for all FIB, electroplated, annealed, and Ar plasma-treated pillars as compared to a range of theoretical strengths and the yield strength of bulk gold. 

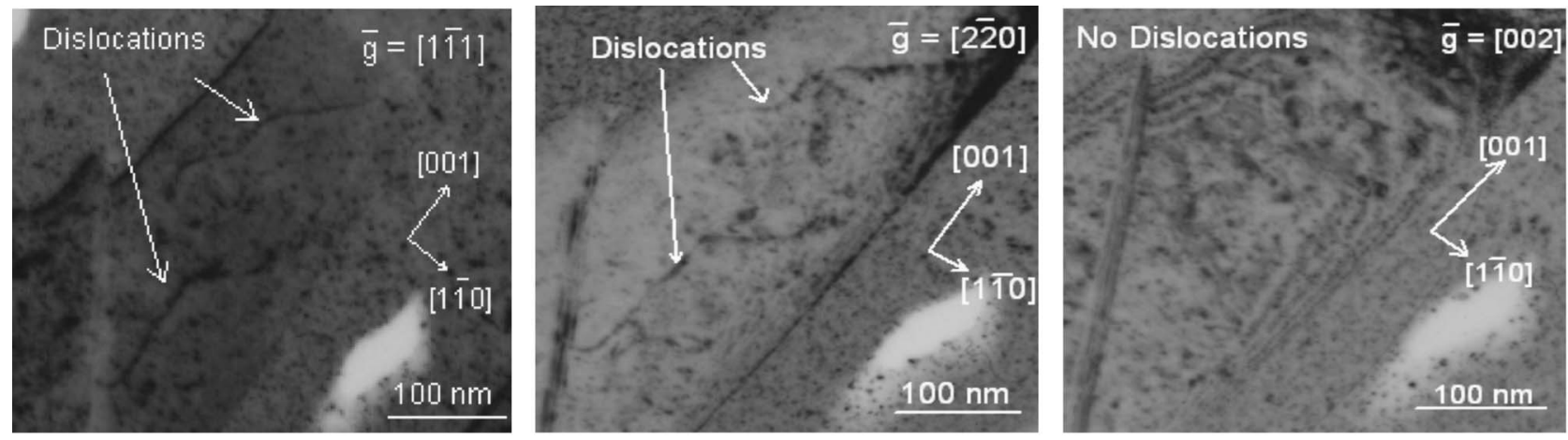

FIG. 5. Negatives of weak-beam dark field TEM images of a deformed pillar at the [110] zone axis. Two dislocation lines are clearly present for the $\bar{g}=[1 \overline{1} 1]$ and $\bar{g}=[2 \overline{2} 0]$ diffraction vectors and disappear for the $\bar{g}=[002]$. The negatives taken on a transparent film reverse the polarity of the colors.

present on the surface of the pillars, it is not a major contributing factor in the strength increase.

One possible explanation for these high strengths can be developed using the concept of dislocation starvation. In ordinary plasticity, dislocation motion leads to dislocation multiplication by the double cross-slip, invariably leading to softening before strain hardening occurs through elastic interaction of dislocations. Unlike in bulk samples, dislocations in sub-micron-sized crystals can travel only very small distances before annihilating at free surfaces, thereby reducing the overall dislocation multiplication rate. Gliding dislocations leave the crystal more rapidly than they multiply, decreasing the overall dislocation density. Such processes would lead to a dislocation-starved state requiring very high stresses to nucleate new dislocations. Our phenomenological model describes this hypothesis by calculating the evolving dislocation density in the course of deformation. All equations and relevant details of this model as well as the model's predictions clearly showing the differences in deformation mechanism between a relatively large and a relatively small pillar are shown in Ref. 19(b). These theoretical results also agree well with the experimental data shown in Fig. 3. The dislocation starvation model we give here focuses only on the starvation process and does not incorporate any possible dislocation nucleation events. The modeling simply shows that for crystals below a critical size the dislocation density should decline in the course of deformation and tend toward a dislocation-starved state. When such a state is reached, the stress would be expected to rise abruptly leading subsequently to the nucleation of new dislocations at the everhigher nucleation stresses. This increase in the dislocation nucleation stresses may be attributed to the scarcity of sources in smaller pillars. An in-depth computational dislocation dynamics analysis is required to fully assess the dislocation behavior in a confined-geometry sample under the described conditions. Some promising attempts have already been made to create a model explaining the presence of a characteristic length scale in mechanical deformation. ${ }^{14-16}$

Transmission electron microscopy (TEM) observations of pillar cross sections before and after deformation were made in an effort to check one of the main predictions of the dislocation starvation theory. Due to the very small dimensions of the pillar specimen, conventional TEM preparation tech- niques could not be utilized. The preparation of the electrontransparent samples involved the application of the Omniprobe capability in the focused ion beam. Since the pillar was exposed to the $\mathrm{Ga}^{+}$ion beam throughout this sample preparation process, several attempts were made to clean the surface of TEM samples in the precision ion polishing system (PIPS). The Gatan PIPS ion mill is equipped with two low-energy $\mathrm{Ar}^{+}$plasma beams whose tilt positions can be adjusted to any value between $0^{\circ}$ and $10^{\circ}$. While this method of surface preparation is usually very effective, it did not prove to be so in this case due to the slight misalignment between the pillar and the TEM grid. This misalignment leads to an error in the calculation of the plasma beam angles, resulting in either milling away a part of the pillar or not removing any damage. TEM images of a deformed pillar cross section along the [110] zone axis are shown in Fig. 5. The pillar was compressed in the [001] direction, which is also shown in the figure.

In the TEM image, two dislocation lines which are clearly present for the [1 $1 \overline{1} 1]$ and [2 $\overline{2} 0]$ diffraction vectors disappear for the $\bar{g}=[002]$ case. TEM images were taken at two different zone axes, and two sets of invisibility conditions were determined in order to calculate the Burgers vector of the featured dislocations. Based on this analysis, the Burgers vector of both dislocations visible in the TEM images is [1ํㅣ, as shown in Fig. 5. As expected, this Burgers vector is perpendicular to the loading axes of the pillar [001]. The transmission electron microscopy images of the deformed pillars are found to be void of any mobile dislocations and show only two dislocations whose Burgers vectors are perpendicular to the loading axis and thus not driven by the applied stress. This supports the argument that all of the mobile dislocations escaped from the crystal, leaving it only with these two nonmoving dislocations that must have formed through a reaction of previously present mobile dislocations. TEM sample preparation inevitably leaves residual FIB damage on the specimen surface. For example, the surface layer on the $80 \mathrm{~nm}$ thick TEM foil is approximately $5 \mathrm{~nm}$ thick and manifests itself as a collection of small dislocation loops. The appearance of these loops in the TEM images for both undeformed and deformed cases indicates that they are most likely due to the FIB damage during sample preparation and are not produced by deformation. 
The existence of these dislocations on the surface of TEM samples brings to question the possibility of their presence in the original pillar surfaces, which might hinder the motion of gliding dislocations as they are leaving the crystal. It should be noted, however, that in the course of TEM sample preparation, the foil is subjected to higher doses of $\mathrm{Ga}^{+}$current compared to the actual pillar fabrication, due to the nature of the incident ion beam angles. TEM sample preparation requires varying the angle between the sample and the ion beam, resulting in more surface damage than during the FIB preparation, where the ion beam is strictly orthogonal to the pillar top and parallel to the sides of the pillar. Therefore, it would be expected for the TEM-transparent samples to contain significantly more dislocation loops than the surface of a FIB-machined pillar. Nonetheless, the possible presence of some FIB damage on the surfaces of test samples is a complication that requires further study.

According to the dislocation starvation hypothesis described above, mobile dislocations are thought to escape from the crystal at the nearest free surface before multiplying and interacting with other dislocations. Such processes lead to a dislocation-starved state, and nucleation of new dislocations in the newly-formed perfect crystal is required to accommodate further plastic deformation. When a perfect crystal is subjected to an applied stress, the activation energy required for nucleation of dislocation loops is a strongly decreasing function of the ratio of the applied shear stress and the ideal shear strength, as shown by $\mathrm{Xu}$ and Argon. ${ }^{21}$ The stress levels attained for our smallest pillars correspond to $\sim 0.45 \tau_{\text {theoretical }}$, requiring a high energy of $\sim 13 \mathrm{eV}$ to homogeneously nucleate a dislocation loop. Theoretical shear strength values in this previous work, however, do not take into account the atomistic nature of nucleating a dislocation near a free surface, where the values of theoretical strength are expected to be lower. Therefore, in the presence of free surfaces, the fraction of applied stress to the theoretical strength will be higher, requiring lower, more realistic energies to activate nucleation. In order to explore the possibility of a nucleation-controlled deformation mechanism, it is useful to compare our experimental results to those obtained computationally.

Several promising studies have been recently published addressing the observed size effect at the nanoscale. In the molecular dynamics work of Horstemeyer et al. ${ }^{14}$ the em- bedded atom method (EAM) is employed to assess length scale behavior during shear deformation of a fcc crystal. These atomistic simulations reveal that dislocation nucleation at free surfaces is the mechanism of yielding in pristine crystals. The following power law dependence was reported by this study: $\tau_{\text {rss }} / \mu=3.2 \times 10^{-5}\left(V / A_{\mathrm{s}}\right)^{-0.38}$, where $\tau_{\text {rss }}$ is the resolved shear stress in the slip plane, $\mu$ is the shear modulus of $\mathrm{Au}$, and $V$ and $A_{s}$ are the volume and the surface area, respectively. Here, the volume is normalized by a constant length in order to force the quantity on the right-hand side to be dimensionless. Deshpande et al. ${ }^{15}$ performed dislocation dynamics simulations of a single crystal in compression and in tension. In this model, plastic flow was found to arise from the collective motion of discrete dislocations that nucleate from fixed Frank-Read sources which are activated both by the applied stress and the stress fields of nearby gliding dislocations. The authors also derived a power law expression for the flow stress as a function of the lateral dimensions of the sample, $W, \sigma_{f}=67 \times 10^{-5}\left(W / W_{0}\right)^{-0.49}$, where the leading constant of 67 has units of MPa. Incipient plasticity was also investigated by Zuo et al. in $2005 .{ }^{16}$ These authors also employed EAM to compute atomic fluctuations leading to dislocation nucleation in a small computational cell of $\mathrm{Ni}_{3} \mathrm{Al}$ subjected to homogeneous shear. It was found that atoms with relative displacements larger than $\frac{1}{2}$ of the Shockley partial vector formed "hot zones," resulting in the creation of partial dislocation loops. Fitting our gold data to this model required modification of two parameters, which are functions of the strain rate and elastic properties of the deformed material. The graphs representing predictions of these models ${ }^{14-16}$ in comparison to the present experiments are shown online [Ref. 19(c)]. While the overall trends of these models compare favorably with experimental data, the inevitable idealizations associated with computational studies and large differences in sample sizes may be responsible for some of the differences in the predicted mechanical behavior.

The authors gratefully acknowledge financial support of this project through grants provided by a NSF-NIRT grant (CMS-0103257) and the Department of Energy (DE-FG0389ER45387). We especially would like to thank W. Oliver (MTS Corporation), M. Uchic (AFRL), as well as A. Marshall, D. Pickard, G. Feng, E. Perozziello, B. Jones, and J. Cheng (Stanford University) for their help in this work.
${ }^{1}$ Y. Wei and J. W. Hutchinson, J. Mech. Phys. Solids 51, 2037 (2003).

${ }^{2}$ J. S. Stolken and A. G. Evans, Acta Mater. 46, 5109 (1998).

${ }^{3}$ W. D. Nix and H. Gao, J. Mech. Phys. Solids 46, 411 (1998).

${ }^{4}$ H. D. Espinosa and B. C. Prorok, J. Mater. Sci. 38, 4125 (2004).

${ }^{5}$ L. Nicola, E. Van der Giessen, and A. Needleman, J. Appl. Phys. 93, 5920 (2003).

${ }^{6}$ Q. Ma and D. R. Clarke, J. Mater. Res. 10, 853 (1995).

${ }^{7}$ See, for example, N. A. Stelmashenko, M. G. Walls, L. M. Brown, and Y. V. Millman, Acta Mater. 41, 2855 (1993); Y.
Huang, Z. Xue, H. Gao, W. D. Nix, and Z. C. Xia, J. Mater. Res. 15, 1786 (2000).

${ }^{8}$ E. Arzt, Acta Mater. 46, 5611 (1998).

${ }^{9}$ D. Y. Yu and F. Spaepen, J. Appl. Phys. 95, 2991 (2003).

${ }^{10}$ W. D. Nix, Metall. Trans. A 20A, 2217 (1989).

${ }^{11}$ E. Arzt, G. Dehm, P. Gumbsch, O. Kraft, and D. Weiss, Prog. Mater. Sci. 46, 283 (2001).

${ }^{12}$ W. W. Gerberich, J. C. Nelson, E. T. Lilleodden, P. Anderson, and P. J. T. Wyrobek, Acta Mater. 44, 3585 (1996).

${ }^{13}$ S. S. Brenner, J. Appl. Phys. 27, 1484 (1956); S. S. Brenner, R. 
Doremus, B. W. Roberts, and D. Turnbull, Growth and Perfection of Crystals (Wiley, New York, 1958), p. 157.

${ }^{14}$ M. F. Horstemeyer, M. I. Baskes, and S. J. Plimpton, Acta Mater. 49, 4363 (2001).

${ }^{15}$ V. S. Deschpande, A. Needleman, and E. Van der Giessen, J. Mech. Phys. Solids (to be published).

${ }^{16}$ L. Zuo, A. H. W. Ngan, and G. P. Zheng, Phys. Rev. Lett. 94, 095501 (2005).

${ }^{17}$ M. D. Uchic, D. M. Dimiduk, J. N. Florando, and W. D. Nix, Science 305, 986 (2004).

${ }^{18}$ J. R. Greer, W. C. Oliver, and W. D. Nix, Acta Mater. 53, 1821
(2005); J. R. Greer, Ph.D. dissertation, Stanford University (2005).

${ }^{19}$ See EPAPS Document No. E-PRBMDO-73-061624 for (a) full Auger analysis, (b) dislocation starvation mechanism, and (c) comparison of computational studies with our experimental data. This document can be reached via a direct link in the online article's HTML reference section or via the EPAPS homepage (http://www.aip.org/pubservs/epaps.html).

${ }^{20}$ S. Ogata, J. Li, N. Hirosaki, Y. Shibutani, and S. Yip, Phys. Rev. B 70, 104104 (2004).

${ }^{21}$ G. Xu and A. S. Argon, Philos. Mag. Lett. 80, 605 (2000). 